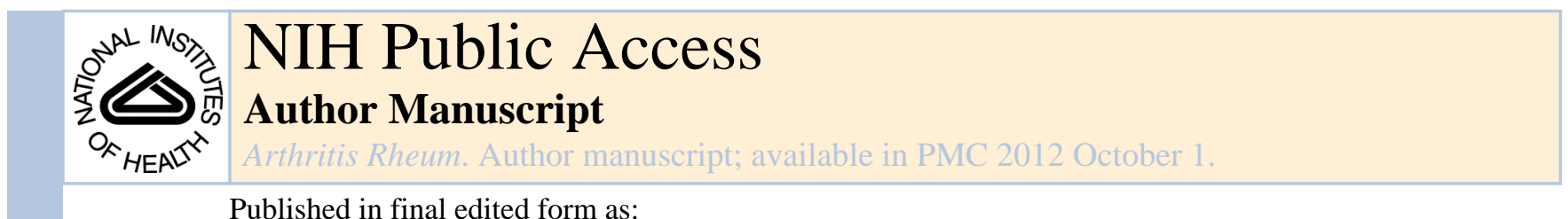

Published in final edited form as:

Arthritis Rheum. 2011 October ; 63(10): 2848-2850. doi:10.1002/art.30505.

\title{
Biosimilars: The Debate Continues
}

Robert A. Colbert, MD, PhD[Senior Investigator] and

Chief, Pediatric Translational Research Branch, NIAMS/NIH, 10 Center Drive, CRC, Rm 1-5142, Bethesda, Maryland 20892

Bruce N. Cronstein, MD[Paul R. Esserman Professor of Medicine]

NYU School of Medicine, 550 First Ave., NBV16N1, New York, New York 10016

\section{COMMENTARY}

There is no question that biologics have had a major impact in rheumatology as in other areas of medicine, with many more potentially efficacious drugs in various stages of development. However, the high cost of biologics is a growing concern for consumers and insurers, particularly as new products become available and their use for the treatment of autoimmune and autoinflammatory diseases continues to expand. Worldwide, an estimated $\$ 130 \mathrm{~B}$ was spent on biologics in 2009, with $\$ 6.6 \mathrm{~B}$ in sales of Rituxan (Rituximab) alone in 2010 (1). Biosimilars (also referred to as follow-on biologics) are intended to be subsequent versions of reference biologics with comparable safety and efficacy profiles, analogous to generics of brand name drugs. Biosimilars become potential cost-saving alternatives once patent and exclusivity rights on reference biologics have expired. They also represent a multi-billion dollar market for producers, and a threat to innovators who produce the reference biologics, as they will undoubtedly experience substantial losses in market share. The stakes are enormous.

In 1984 the U.S. Congress approved the Drug Price Competition and Patent Term Restoration Act (also known as the Hatch-Waxman Act), establishing an abbreviated new drug application (ANDA) pathway for generics. The main difference between a new drug application and an ANDA for a generic, is that the latter does not require pre-clinical and clinical studies if bioequivalence - the rate and extent of absorption - can be demonstrated. This saves time and considerable expense in the approval process. Generic equivalents of many drugs have proven to be relatively straightforward to produce, and consequently the use of generics has risen dramatically in the last two decades, with consumer savings estimated to be about $\$ 10 \mathrm{~B}$ a year in 2004. Can a similar abbreviated approval pathway be used for biosimilars to reap comparable benefits for consumers and insurers? This is where the debate begins.

\section{Disclosures}

Dr. Colbert has nothing to disclose.

Dr. Cronsteindiscloses the following:

Intellectual Property. Dr. Cronstein holds or has filed applications for patents on the use of adenosine $\mathrm{A}_{2 \mathrm{~A}}$ receptor agonists to promote wound healing and use of $\mathrm{A}_{2} \mathrm{~A}$ receptor antagonists to inhibit fibrosis; use of adenosine $\mathrm{A}_{1}$ receptor antagonists to treat osteoporosis and other diseases of bone; the use of adenosine $A_{1}$ and $A_{2 B}$ Receptor antagonists to treat fatty liver, and; the use of adenosine $\mathrm{A}_{2} \mathrm{~A}$ receptor agonists to prevent prosthesis loosening

Consultant Relationships (within the past two years). King Pharmaceutical (licensee of patents on wound healing and fibrosis noted above). CanFite Biopharmaceuticals, Savient Pharmaceuticals, Bristol-Myers Squibb, Roche Pharmaceuticals, Cellzome, Tap (Takeda) Pharmaceuticals, Prometheus Laboratories, Regeneron (Westat, DSMB), Sepracor, Amgen, Endocyte, Protalex, Allos, Inc., Combinatorx, Kyowa-Hakka (Kirin), Bristol-Myers Squibb, Novartis. 
In most instances 'biologics' refers to a class of medications produced by living cells using recombinant DNA technology (2). The recombinant DNA is introduced into the cells, which have the machinery to read and decode the DNA, and produce complex proteins that can be purified and used for medicinal purposes. Typical biologics include proteins that are intended to be almost identical to natural products that the body makes, often used as replacement therapy in the case of a genetic deficiency, or simply to augment the body's response to an endogenous product. Other biologics include monoclonal antibodies that bind to soluble or cell surface proteins and block overactive pathways or cells, or creatively engineered proteins that mimic receptors or naturally occurring receptor antagonists, but are modified to be soluble or more stable, and thus prevent activation of signaling pathways.

The problem arises when one tries to develop a copy, or biosimilar, of a reference biologic. The challenge is quite different from reproducing small chemical compounds to produce a generic drug. The molecular and structural complexity of most biologics makes it difficult if not impossible to produce exact replicas. Even if the same recombinant DNA sequence is used, the composition of the final product is influenced by a variety of factors including the cells used to produce the protein, culture conditions, post-translational modifications, purification methods, stabilization and storage conditions, and packaging. Manufacturers of reference products may use proprietary growth and purification conditions, and therefore knowing the exact sequence of the DNA construct used to produce the protein is not sufficient to produce the same biologic product. What may seem to be subtle differences between a biosimilar and the reference biologic can affect efficacy as well as safety and immunogenicity.

In March 2010 the Patient Protection and Affordable Care Act, more commonly known as Health Care Reform, was signed into law in the U.S. It outlines an abbreviated approval pathway for biological products that are demonstrated to be "highly similar" biosimilar) to, or "interchangeable" with, an FDA-licensed biological product. Known as the Biologics Price Competition and Innovation Act (BPCIA), the BPCIA is similar to the 1984 Drug Price Competition and Patent Term Restoration Act that established an abbreviated approval pathway for generics. Currently, according to the FDA definition (3), a product is biosimilar if data from analytical stud ies, animal studies, and a clinical study or studies, demonstrates the product to be 'highly similar' to the reference product, notwithstanding minor differences in clinically inactive components, and if there are no clinically meaningful differences in terms of safety, purity and potency. To meet the higher standard of interchangeability requires demonstrating that the product can be expected to produce the same clinical result as the reference product in any given patient, and, if the biologic is administered repeatedly, there is no greater risk in terms of safety or diminished efficacy from alternating or switching than there would be from continued administration of the reference product. If interchangeability has been demonstrated, then thepharmacist can substitute the biosimilar for the reference product without the intervention of the prescribing health care provider. At issue are the nature and quantity of evidence needed to establish biosimilarity and interchangeability for biologics. In the U.S. the bar seems to be set pretty high.

In November 2010 the FDA held a public hearing to obtain input from various stakeholders on how to implement the BPCIA (4). The results were less than shocking considering the financial and health care implications. Innovators who have developed and produce the reference biologics think the bar should remain high, while companies who produce generics favor lower clinical standards to demonstrate biosimilarity. Patient advocacy organizations and medical specialty professional societies insist, understandably, that safety and efficacy be top priorities. While it should be emphasized that everyone advocates safety and efficacy for biosimilars, the perceptions of how much testing should be required before a drug is 
marketed vary dramatically. Other issues received attention at the FDA hearing, including the question of whether a biosimilar of a reference biologic approved for more than one indication, would need to be tested in each of those indications, and whether the clinical target should be equivalence or non-inferiority.

While the debate goes on in the U.S., European regulatory authorities have continued to move forward. The European Medicines Agency (EMA) was the first to develop a comprehensive approach to licensing biosimilar agents (http://www.ema.europa.eu/). EMA has developed a flexible case-by-case approach to licensing biosimilars with clear and precise regulatory tracks for licensing of specific agents such as growth hormone, insulin and filagrims. Pre-clinical data that establish the similarity of the biosimilar to the reference biologic with respect to antigen or ligand binding, evidence of the identity of the agents (but not the glycosylation state in the case of larger proteins) and results of appropriately designed pre-clinical pharmacokinetic and pharmacotoxicologic tests in animals are required. In addition, regulators scrutinize manufacturing standards. Requirements for demonstration of pharmacokinetics and pharmacodynamics in humans are also necessary, but these requirements are eclipsed by the need to demonstrate similar clinical efficacy and safety of the biosimilar compared to that of the reference biologic. For a number of proteins regulatory pathways have been established with clinical trial requirements ranging from none (for human insulin preparations) to trials comparing hundreds of patients taking either the original biologic or the biosimilar.

Because of the structural complexity of biologics a considerable problem for establishing an abbreviated regulatory path for biosimilars is immunogenicity. One example where this has had significant consequences is the development of pure red cell aplasia in patients receiving recombinant erythropoietin. Although this may be a rare occurrence, there is a formal requirement for an extended pharmacovigilance plan that must be approved and in place. The requirement for pharmacovigilance dictates that a single generic name cannot be applied to the reference biologic and subsequent biosimilar agents, since it would not be possible to detect a novel toxicity of the biosimilars. The issue of approval for single vs. multiple indications and whether additional clinical testing will be required also remains to be resolved. This will likely be handled on a case-by-case basis. Nonetheless, a number of biosimilar agents have already been licensed in Europe (although none for rheumatology indications) following the regulatory guidance offered by EMA (1). In addition, the World Health Organization recently issued a guidance document for licensing of biosimilars, as have Korea and Singapore. Canada, Australia and Japan are now finalizing regulatory guidance documents and it is likely that most other countries will follow suit rapidly. Most of the regulatory pathways adopted to date are similar.

The EMA has just made another step forward with guidelines for biosimilar monoclonal antibodies (5). Their Committee for Medicinal Products for Human Use (CHMP) of the EMA has said that while comparative Phase III clinical studies demonstrating safety and efficacy for biosimilars are necessary, they have placed the focus clearly on designing trials to establish 'similarity' to the reference compound without necessarily demonstrating patient benefit. The guidelines advise using selected patient populations where the biologic is known to be highly efficacious to look for similar effects of the biosimilar. Differences would be expected to be more readily apparent in smaller numbers of individuals. The use of more homogeneous patient populations with similar disease severity and a lack of comorbidities would further facilitate these comparisons and keep sample sizes down, thus minimizing cost. In establishing these guidelines the CHMP/EMA have established a lower, yet quite rational, hurdle for establishing monoclonal antibody biosimilarity. The new guidelines also begin to address the issue of multiple indications. They outline situations where extrapolation of clinical efficacy and safety data for another indication may be 
possible, such as when the mechanism of action of the biologic is the same. When the mechanism of action for two or more indications is different, then the situation will be more complex and require additional testing. It should be noted that these guidelines are currently open for comment, and are likely to be further revised prior to their general acceptance.

The American College of Rheumatology through the Committee on Research has developed a position statement on biosimilars. In essence, it puts the patient first, emphasizing that safety and efficacy are paramount. However, it can be argued that unless biosimilars are much less expensive than their reference biologics, the entire exercise is academic. Decisions about biosimilarity and interchangeability need to be data driven, but the process needs to recognize the heterogeneity of different classes of biologics with flexibility built into the quidelines. For biosimilars to reduce the cost of health care and increase the availability of effective treatments will require that they are significantly less expensive than reference biologics, and that they are prescribed with confidence. Given issues related to immunogenicity, interchangeability may be one of the highest hurdles to clear. Stay tuned; the debate is far from over.

\section{Acknowledgments}

\section{Support}

This work was supported by the National Institutes of Health (NIH). Dr. Colbert received support from the National Instituteof Arthritis and Musculoskeletal and Skin Diseases (NIAMS) Intramural Research Program (AR041184). Dr. Cronstein was supported by NIH grants AR56672, AR56672S1, and AR54897, andthe NYU-HHC Clinical and Translational Science Institute (UL1RR029893).

\section{References}

1. Carey K. Biosimilars encircle Rituxan, US debates innovator exclusivity. Nat Biotech. 2011; 29:177.

2. Jelkmann W. Biosimilar epoetins and other "follow-on" biologics: Update on the European experiences. Amer J Hematol. 2010; 85:771. [PubMed: 20706990]

3. Department of Health and Human Services, Food and Drug Administration, Docket No. FDA-2010$\mathrm{N}-0477$

4. Mullard A. Hearing shines spotlight on biosimilar controversies. Nat Rev Drug Disc. 2010; 9:905.

5. Similarity trials. Nat Biotech. 2011; 29:1. 